\title{
Adaptive Cancellation of Power-line Interference from Biopotential Measurement
}

\author{
Yue-Der Lin, Hui-Hsun Huang and Fok-Ching Chong \\ Institute of Electrical Engineering, National Taiwan University, Taiwan
}

\section{ABSTRACT}

Power-line interference is a common problem during biopotential measurement. Here an easily implemented algorithm for adaptive power-line interference canceling is presented. The proposed algorithm is based on the iterative update of amplitude, frequency and phase of a sine wave according to the least-mean-squares (LMS) algorithm. The advantage of the proposed technique is no extra reference signal needed during the biopotential measurement.

Keywords: Power-line interference, leastmean-squares (LMS) algorithm.

\section{INTRODUCTION}

Interference from power lines is the main noise source in biopotential measurement. Although the high input impedance and high CMRR instrumentation amplifier, the drivenright-leg (DRL) circuit [1] and the isolation of signal and power [2] have been proposed for the construction of biopotential amplifier to eliminate such an interference, power-line noise may appear as normal mode signals, owing to the potential-divider effect [3] and the non-ideal characteristics of active and passive components.

In earlier applications, notch filter have usually been used to remove remaining interference. However, the frequency of the power-line noise is not always stable at exactly $60 \mathrm{~Hz}$ (or $50 \mathrm{~Hz}$ ). The filtering performance will degenerate as the power-line frequency deviates from the notch frequency. Self-tuned notch filter that can track the deviation of power-line frequency is an attractive approach [4], but additional devices are needed which may increase circuit size and cost (especially on multichannel recording). Another promising approach is the application of adaptive filter. The typical adaptive noise canceling (ANC) technique requires an extra reference input containing the interference for removing the interference component from the contaminated signal [5]. This will need additional amplifier and analog-to-digital converter to record the interference signal. Recently, So proposed a novel adaptive algorithm used for sinusoidal interference removal [6]. In his method, no reference is needed, but the frequency of the sinusoidal interference should be a fixed value.
In this short paper, So's method is modified to allow the deviation of interference frequency. Experimental result of contaminated electrocardiogram (ECG) is given to demonstrate the feasibility of the proposed algorithm.

\section{METHOD}

Let the measured signal be denoted as $r(n)$, and

$r(n)=s(n)+\alpha(n) \cos \left[\frac{2 \pi f(n) n}{f_{S}}+\phi(n)\right]$, where $s(n)$ is the desired signal, $f S$ is the sampling frequency, while $\alpha(n), f(n)$ and $\phi(n)$ represent the unknown amplitude, frequency and phase, respectively, of the interference signal at the time index $n$. The principle of the algorithm is to update $\alpha(\cdot)$, $f(\cdot)$ and $\phi(\cdot)$ iteratively. When they converge to the same values as those of the interference signal, The desired signal $S(\cdot)$ can be retrieved by subtracting the sinusoidal signal characterized by $\alpha(\cdot), f(\cdot)$ and $\phi(\cdot)$ from $r(\cdot)$.

Let

$e(n)=r(n)-\hat{\alpha}(n) \cos \left[\frac{2 \pi \hat{f}(n) n}{f s}+\hat{\phi}(n)\right]$, where $\hat{\alpha}(n), \hat{f}(n)$ and $\hat{\phi}(n)$ denote the estimates of $\alpha(n), f(n)$ and $\phi(n)$, respectively. Then the mean-squared error is

$J(n)=E\left[\|e(n)\|^{2}\right]$

Based on the LMS algorithm, we can derive $\hat{\alpha}(n+1)=\hat{\alpha}(n)-\frac{1}{2} \mu_{\alpha} \frac{\partial J(n)}{\hat{\partial}(n)}$

$$
=\hat{\alpha}(n)+\mu_{\alpha} e(n) \cos \left[\frac{2 \pi \hat{f}(n) n}{f s}+\hat{\phi}(n)\right]
$$

$\hat{f}(n+1)=\hat{f}(n)-\frac{\mu_{f}}{2 \hat{\alpha}(n) \pi_{n}} \frac{\partial J(n)}{\partial \hat{f}(n)}$

$$
=\hat{f}(n)-\frac{2 \mu_{f} e(n)}{f s} \sin \left[\frac{2 \pi \hat{f}(n) n}{f_{S}}+\hat{\phi}(n)\right]
$$

and 


$$
\begin{aligned}
\hat{\phi}(n+1) & =\hat{\phi}(n)-\frac{\mu,}{2 \hat{\alpha}(n)} \frac{\partial J(n)}{\partial \hat{\phi}(n)}, \\
& =\hat{\phi}(n)-\mu_{,} e(n) \sin \left[\frac{2 \pi \hat{f}(n) n}{f s}+\hat{\phi}(n)\right]
\end{aligned}
$$

where $\mu_{\alpha}, \mu_{f}$ and $\mu_{\phi}$ are positive real-valued constants that determine convergence rate and stability of the algorithm.

The summary of the proposed algorithm is listed in Table I.

\section{RESULT}

Fig. 1 shows one of the experimental results for the proposed algorithm. In this figure, Fig.1(a) is the ECG signal contaminated by $60-\mathrm{Hz}$ interference, Fig.1(b) is the recovered ECG signal, and Fig.1(c) is the original ECG signal. The initial values of amplitude, phase and frequency are $0.25,0$ and 58 , respectively, The step-size parameters are: $\mu_{\alpha}=0.02, \mu_{f}=0.005$ and $\mu_{\phi}=0.005$.

From this result, we can find the sinusoidal interference can be removed asymptotically from the contaminated ECG signal without causing ECG waveform distortion.

\section{CONCLUSION}

An LMS-style algorithm that can eliminate sinusoidal interference using the update of amplitude, frequency and phase parameters is developed. The theoretical analysis is given and the algorithm is verified by the experiment of ECG signal. The result demonstrates that the proposed algorithm can be successfully applied to the elimination of $60-\mathrm{Hz}$ interference in contaminated ECG signal.

\section{REFERENCE}

[1] B. B. Winter and J. G. Webster, "Drivenright-leg circuit design", IEEE Trans. Biom. Eng., 1983, Vol.30, No.1, pp.62-66.

[2] A. C. Metting van Rijn et al., "The isolation mode rejection ratio in bioelectric amplifiers", IEEE Trans. Biom. Eng., 1991, Vol.38, No.11, pp.1154-1157.

[3] J. C. Huhta and J. G. Webster, "60-Hz interference in electrocardiography", IEEE Trans Biom. Eng., 1973, Vol.20, No.2, pp.91-101.

[4] S. K. Yoo et al., "Simple self-tuned notch filter in a bio-potential amplifier", Med. \& Biol. Eng. \& Comp., March 1997, Vol.35, pp.151-154.

[5] B. Widrow et al., "Adaptive noise canceling: Principles and applications", Proc. IEEE, 1975, Vol.63, pp.1692-1716.

[6] H. C. So, "Adaptive algorithm for sinusoidal interference cancellation", Elec. Lett., 1997, Vol.33, No.22, pp.1910-1912.

Table I The proposed algorithm. Initialization : $\alpha(1), f(1)$ and $\phi(1)$.

Given : $r(\cdot)=$ The contaminated signal.

$\mu_{\alpha}, \mu_{f}$ and $\mu_{\phi}=$ Step-size parameters.

Computation :

$$
e(1)=r(1)-\alpha(1) \cos \left[\frac{2 \pi f(1)}{f s}+\phi(1)\right],
$$

for $\mathrm{i}=1$ to $\mathrm{N}-1(\mathrm{~N}$ : data length of $r(\cdot)$.)

$$
\begin{aligned}
& \alpha(i+1)=\alpha(i)+\mu_{\alpha} e(i) \cos \left[\frac{2 \pi f(i) i}{f s}+\phi(i)\right], \\
& \phi(i+1)=\phi(i)-\mu_{\phi} e(i) \sin \left[\frac{2 \pi f(i) i}{f s}+\phi(i)\right], \\
& f(i+1)=f(i)-2 \mu_{i} e(i) \sin \left[\frac{2 \pi f(i) i}{f s}+\phi(i)\right], \\
& e(i+1)=r(i+1)-\alpha(i+1) \cos \left[\frac{2 \pi f(i+1)(i+1)}{f s}+\phi(i+1)\right] .
\end{aligned}
$$

end

(a)
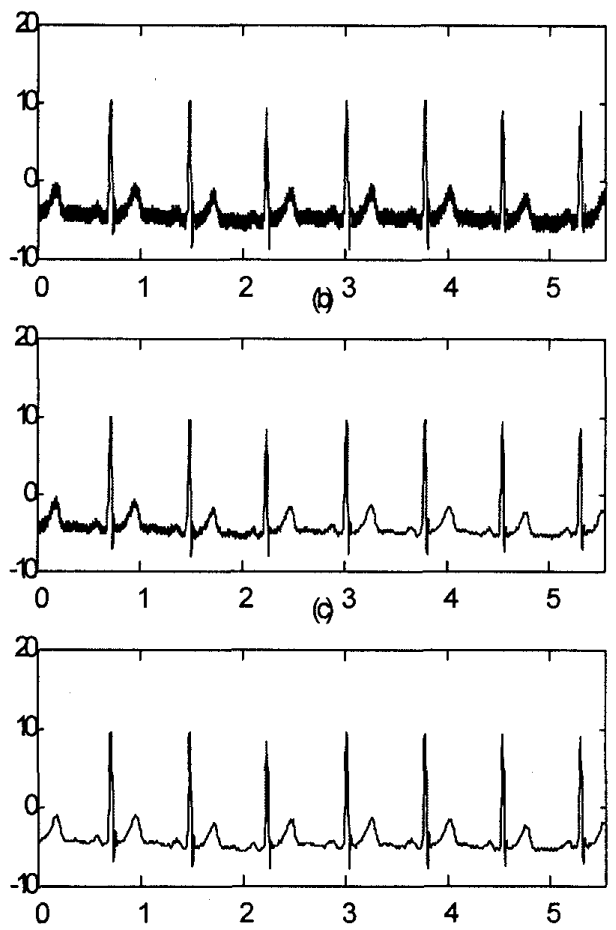

Fig.1 The experimental result. The $x$-axis is in second unit, while the $\mathrm{y}$-axis is in $\mathrm{mV}$ unit. The sampling frequency is $360 \mathrm{~Hz}$. 